\title{
Téoros
}

Revue de recherche en tourisme

\section{Des agences de voyages de plus en plus responsables}

\section{Louis Jolin}

Volume 9, numéro 3, novembre 1990

La distribution du produit touristique

URI : https://id.erudit.org/iderudit/1079888ar

DOI : https://doi.org/10.7202/1079888ar

Aller au sommaire du numéro

Éditeur(s)

Université du Québec à Montréal

ISSN

0712-8657 (imprimé)

1923-2705 (numérique)

Découvrir la revue

Citer cet article

Jolin, L. (1990). Des agences de voyages de plus en plus responsables. Téoros, 9(3), 28-30. https://doi.org/10.7202/1079888ar d'utilisation que vous pouvez consulter en ligne.

https://apropos.erudit.org/fr/usagers/politique-dutilisation/ 


\section{Des agences de voyages de plus en plus responsables}

La protection des clients des agences de voyages fut l'objectif visé par l'adoption de plusieurs lois dans les pays occidentaux. Outreces lois particulières, les grands principes du droit commun furent mis à contribution par les tribunaux pour régler les litiges sans cesse croissants entre des consommateurs avertis et leurs agences de voyages. La responsabilité des agents de voyages est de plus en plus affirmée, bien que des nuances s'imposent et que l'évolution des technologies d'informations pourrait inverser la tendance.

\section{La Loi sur les agents de voyages: portée et limites}

Depuis plus de quinze ans, les agents de voyages du Québec sont soumis aux dispositions de la Loi sur les agents de voyages". A la suite de nombreux abus au cours des années ' 60 et au début des années '70 (fraudes, faillites, etc.), cette loi, adopteeen 1974, visait à assainir la pratique commerciale des agents de voyages tout en protégeant le touriste consommateur. Elle fut modifiée en 1977, 1979, 1981 et chaque fois pour accentuer son caractère de protection du consommateur. En 1981 d'ailleurs, la loi passa sous la responsabilité du ministre de l'Habitation et de la protection du consommateur et, plus spécifiquement, de l'Office de protection du consommateur.

Un règlement d'application ${ }^{2}$ précise les dispositions générales de la loi. Pour l'essentiel, il remonte à 1977 , mais il fut amendé à plusieurs reprises, les dernières modifications d'importance sont récentes et datent du Décret 449-90 du 4 avril 19903. Ces modifications (en vigueur à compter du 30 avril 1990) amendent dix-neuf articles du règlement qui concernent les modalités de demande et le coût du permis, le compte en fiducie, la publicité faite par un agent de voyages, les divers cautionnements ${ }^{4}$.

La Loi et le Règlement sur les agents de voyages protègent le consommateur de quatre façons: 1. l'obligation pour l'agent de voyages de détenir un permis qui n'est

Monsieut Louis Jolin, directeur de Táoros, est professeur en droit du tourisme au Département. d'études urbaines at touristiques (UOAM). octroyé qu'à certaines conditions relatives à la solvabilité et à l'expérience du demandeur; 2. la mise en place d'un mécanisme visant la protection de l'argent du consommateur (compte en fiducie, cautionnement individuel, cautionnement collectif); 3 . l'obligation pour l'agent de voyages de se conformer à certaines exigences en matière d'information et de publicité; 4. quelques dispositions impératives dans le butd'éviter des mauvaises surprises de dernière heure (délai de remise de documents aux clients, délai d'annulation du voyage...).

Malgré certaines réserves, on peut affirmer que, dans l'ensemble, la loi a atteint son but de protection du consommateur. La force de cette loi réside dans son caractère préventif par l'assainissement de la pratique commerciale et dans le rôle que peut jouer l'Office de protection du consommateur en réglantcertaines plaintes eten entreprenant des actions pour obtenir l'imposition d'amendes aux contrevenants. Ces derniers ont péché surtout aux niveaux de l'exploitation d'une agence sans permis et du non-respect des dispositions concernant le placement en fiducie des sommes versées par les clients ${ }^{5}$.

Mais paradoxalement, cette loi n'est pas d'une grande utilité pour définir la responsabilité d'un agent de voyages à l'égard d'un client insatisfait des prestations obtenues. Les juges $n^{\prime} y$ font que très peu référence sauf pour donner la définition d'un agent de voyages. Les juges ont toujours recours au Code civil et accessoirement, ces derniers temps, à la Loi sur la protection du consommateur ${ }^{6}$.

Avec les années, s'est constituée au Québec une jurisprudence un tant soit peu consistante sur la responsabilité des agents de voyages, mais cette jurisprudence est loin d'être unanime concernant la qualification du contrat qui lie l'agent de voyages à son client et concernant l'étendue de sa responsabilité. Parce que les réclamations ne dépassent pas les quelques milliers de dollars, ce sont le juges de la Cour provinciale (dénommée maintenant la chambre civile de la Cour du Québec) qui ont eu à se prononcer, sur les divers litiges et leurs jugements ne peuvent généralement pas etre portés en appel. La jurisprudence en la matière $\mathrm{n}^{\mathrm{a}} \mathrm{a}$ donc pas été fixée par des jugements de la Cour d'appel du Québec, encore moins de la Cour suprême du Canada. L'admission réccente du recours collectif a permis à la Cour supérieure d'être saisie de quelques litiges et un jugement de cette dernière cour peut être considéré comme un jugement charnière? Il y a peu de doctrine en la matière au Québec: deux auteurs seulement ont véritablement écrit sur laquestion, soit monsieur Roland-Yves Gagné ${ }^{8}$ et madame Thérèse RousseauHoule?. Ces auteurs font aussi souvent référence à la jurisprudence et à la doctrine françaises ${ }^{10}$.

\section{La qualification du contrat et I'étendue de la responsabilité}

Comme le souligne judicieusement Thérèse Rousseau-Houle, "les contrats de voyages ne sont pas toujours de même nature et pour les qualifier, on se heurte à de nombreuses difficultés dues à la variété et à l'extension des fonctions qu'accomplissent les agences de voyages"11. Plusieurs jugements ont souligné qu'il s'agissait d'un contrat de manda ${ }^{12}$, d'autres ont refusé de voir dans

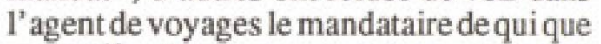
ce soit $t^{13}$. Certains jugements présentent $1^{4}$ agent de voyages comme le mandataire des prestataires de services et des grossistes ${ }^{14}{ }^{4} \mathrm{~d}^{\prime}$ autres comme le mandataire des clients ${ }^{15}$. D'autres jugements ont invoqué les règles du droitcommun relatives à la vente ${ }^{16}$ tandis que quelques jugements semblent y déceler plutốt un véritable con trat d'entreprise $^{117}$. Y a-t-il contradiction? Pas nécessairement, car tout dépend des actes qu'a effectivement posés l'agent de voyages.

Dans la recherche d'un semblant de cohérence au sein de la jurisprudence, les juges et les auteurs ont apporté une distinction fondamentale: l'agent de voyages est-il un simple intermédiaireentre le client et les prestataires de services, ou bien est-il un organisateur de voyages? Cette distinction est contenue dans la définition de l'agent de voyages, à l'article 2 de la Loi sur les agents de voyages, bien que la catégorisation la plus opérationnelle dans cette loi est celle qui discerne le détaillant ( celui qui vend directement au 
public) du grossiste (celui qui traite indirectement avec le public par l'intermédiaire des agents de voyages détaillants). Généralement, l'agent de voyages détaillantn'est qu'un intermédiaire, mais ce n'est pas toujours vrai car il peut organiser réellement un voyage pour le compte de ses clients; le grossiste, quant à lui, est considéré comme un organisateur de voyages. Une fois cette distinction bien établie, les juges ont modulé en conséquence l'étendue de la responsabilité des agents de voyages à l'égard de leurs clients: l'intermédiaire ne sera soumis qu'à une obligation de moyens, l'organisateur de voyages doit assumer une véritable obligation de résultat.

L'agent de voyages, qui n'agit que comme intermédiaire pour vendre un forfaitélaboré par un grossiste, pour retenir des places dans un hôtel ou pour délivrer des billets de transport, doit prouver qu'il a pris les moyens nécessaires, qu'il a fait preuve de diligence et de prudence, qu'il n'a pas commis de faute de son fait personnel.

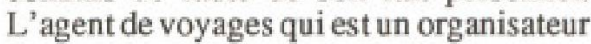
de voyages doit atteindre le résultat; ill ne peut s'exonérer par la preuve d'une simple absence de faute mais doit prouver que la cause du dommage est un cas fortuit.

Cette approche qui vise à moduler l'étendue de la responsabilité selon le fait que l'agent de voyages est considéré comme intermédiaire ou organisateur de voyages renvoie, même si ce n'est pas toujours dit clairement, à la qualification des contrats. L'agent intermédiaire est mandataire des clients ou des fournisseurs de services, incluant le grossiste, ou encore même des deux: selon les dispositions du Code civil, le mandataire doit assumer son mandat avec $\mathrm{l}^{\prime}$ habileté convenable et tous les soins. d'un bon père de famille (art. 1750). Si les clients sont perçus comme les mandants, l'agent n'assume donc à leur égard qu'une obligation de moyens. Si l'agent est considéré comme mandataire du grossiste ou des autres fournisseurs, il n'est pas personnellement responsable envers les tiers, donc les clients (art. 1715). D'après Ethel Groffier ${ }^{18}$, cette analyse n' aboutit pas nécessairement à l'exonération de l'agent de voyages car ce dernier devra identifier aux clients son mandant (grossiste ou transporteur): $s^{\prime}$ il ne le fait pas, sa responsabilité sera engagée.

Quant à l'agent organisateur, les tribunaux ont refusé de le considérer comme intermédiaire entre les clients et les fournisseurs de services (transporteurs, hôteliers) et ont vu l'existence d'un contrat d'entreprise avec les conséquence juridiques qui endécoulent. Interprétant adéquatement la jurisprudence traditionnelle, RolandYves Gagné affirme: "Commeentrepreneur et architecte du voyage, il (1'organisateur) s'engage auprès de son client à coordonner des services, à prévoir des difficultés, à éviter les complications. Tenu de respecter ses engagements, il est soumis à une obligation de résultat"19.

\section{Une tendance plus favorable au consommateur}

Si cela n'a pas toujours été le cas, les juges sont de plus en plus favorables aux clients des agents de voyages. Il y sont aidés notamment par les dispositions de la Loi sur la protection du consommateur.

En effet, comme le souligne Roland Yves Gagné, "le contrat d'organisation de voyages a pour objet un service etest soumis à l'application de la Loi"20. Ainsi, l'article 10 de la Loi sur la protection du consommateur interdit la stipulation par laquelle un commerçant se dégage des conséquences de son fait personnel ou de celui deson représentant. Le jugement de la Cour supérieure dans Comartin c Bordet ${ }^{21}$ va même plus loin: la clause de non responsabilité remise aux clients par l'organisateur le dégageait de toute responsabilité pour les agissements des fournisseurs de services, mais le juge considéra que $l^{2}$ organisateur avait fait preuve de négligence en ne choisissant pas de fournisseurs de services compétents et fiables. Seul un cas fortuit peut en définitive dégager un organisateur de voyages de sa responsabilité.

Dans cet esprit, des arrêtts ont vu l'agent de voyages intermédiaire comme un commerçant vendeur qui peut voir ainsi sa responsabilité engagée comme vendeur. Dans l'affaire Mercier c. Voyages Frontenac Inc. ${ }^{22}$, le juge, bien qu'il ait condamné le grossiste à indemniser le détaillant, condamna le détaillant à payer un certain montant au client parce que le voyage vendu ne correspondait pas à la valeur de ce qui avait été payé.

En se référant à la Loi sur la protection du consommateur, des juges, ces dernières années, ont conclu à la responsabilité de l'agent intermédiaire même si ce dernier avait agien bon père de famille et qu'il avait pris les moyens nécessaires.

Dans la première cause qui reconnut cette responsabilité, Pétrin c. Voyages P.L.M. Inc. ${ }^{23}$, le juge Pierre Durand de la Cour provinciale de Montréal souligne que l'exploitation d'une agence de voyages est une service effectué par un commerçant, donc sujet aux dispositions de la Loi sur la protection du consommateur. $\mathrm{L}^{\mathbf{s}}$ article 41 de ladite loi s"énonçant ainsi: "Un bien ou service fourni doit être conforme à une déclaration ou à un message publicitaire faits a son sujet par le commerçant ou le manufacturier. Une déclaration ou un message publicitaire lie ce commerçant ou ce manufacturier.", le jugeconsidère l'agent devoyages détaillant-intermédiaire comme responsable de la publicité faite par le grossiste et le condamne à dédommager le client pour les inconvênients subis du fait que l'hôtel n'était pas situé sur la plage mais à mille pieds de celle-ci et qu'il n'y avait pas ni piscine, ni terrasse comme l'indiquait la publicité du grossiste. Cette approche basée sur la Loi sur la protection du consommateur contredit, dans ces conclusions, celle qui s'appuie sur le fait que l'agent détaillant est le mandataire du grossiste et représente un changement de direction dans la jurisprudence sur cette question.

Trois années plus tard, en 1986, dans l'affaire Burns c. Vacances Carnival (Québec) Ltée $e^{24}$, le juge Jules Barrière, également de la Cour provinciale de Montréal, apporte une nuance de taille. Pour lui aussi, l'agent de voyages doit être considéré comme un commerçant au sens de la Loi sur la protection du consommateur, mais il ne peut devenir responsable de la publicité du grossiste car "l' obligation de respecter la publicité appartient à celui, du commerçant ou du manufacturier, qui l'a faite ${ }^{\prime \prime}$. Le juge Barrière en arrive à cette conclusion en s'appuyant sur l'article 54 de la loi quiénonce les cas où le consommateur peut recourir contre l'un et l'autre pour le manquement à l'une des obligations résultant des articles 37,38 et 39 , et non de l'article 41. Par ce jugement, le juge Barrière revient a l'approche traditionnelle. Il souligne: "Il est vrai que la Loi sur la protection du consommateur est venue ajouter un fardeau de responsabilité ou de garantie légale aux agents de voyages, mais en pratique, les Cours vont continuer à examiner la situation de fait. Ainsi, si l'agent de woyage agit comme organisateur du voyage, architecte du voyage, il sera soumis à une obligation de résultat, mais s'il agitcomme intermédiaire ou mandataire du consommateur pour acheter un "prêt à partir", il n'aura alors que l'obligation de moyen" ${ }^{n 25}$.

Mais, récemment, le 18 avril 1990, dans un jugement fort élaboré, le juge Jean-Marie Châteauneuf de la lạ Cour du Québec à Trois-Rivières, contredit à son tour le juge Barrière et réhabilite la position du juge Durand. Il s'agit de 1'affaire Gosselin c. Service de voyages Yves Bordeleau Inc. Pour le juge Châteauneuf, 1 'article 41 de la 
Loi sur la protection du consommateur doit être lu" comme signifiant que le commerçant ou le manufacturier ou les deux peuvent être tenus responsables d"une publicité erronée" . La loi impose une obligation de livrer la marchandise vendue (ici le forfait, le "pret-à-partir") sans qu'il soit possible à 1 'agent de voyages détaillant (le vendeur/ commerçant) d'invoquer la conduite fautive du grossiste (assimilé au manufacturier) pour se disculper. Bien sûr, le détaillant pourra toujours par la suite se retoumer contre le grossiste, il conserve son recours récursoire mais, par rapport au client, le détaillant est responsable. De façon explicite, le juge Châteauneuf se démarque de la position traditionnelle, notamment formulée par Roland Yves Gagné pour qui l'agent de voyages intermédiaire n'est tenu qu'à une obligation de moyens.

La Loi sur la protection du consommateur, malgré quelques hésitations jurisprudentielles dans l'interprétation des termes ( ex. le sens de la conjonction "ou" à l'article 41) semble renforcer la responsabilité des agents de voyages détaillants à l'égard de leurs clients. Evidemment, il faudrait attendre les jugements des tribunaux supérieurs pour parler d'une nouvelle jurisprudence définitive sur ce point, mais les faibles montants en jeu dans ce genre de causes ne permettent généralement pas d'aller en appel: il faut plutốt déceler dans les jugements de première instance les nouvelles tendances de plus en plus favorables au consommateur.

D'ailleurs, les positions du juge Durand et du juge Châteauneuf semblent rejoindre la position de la doctrine française actuelle qui reconnaît une responsabilité contractuelle du fait d'autrui. Par cette théorie, il n'est pas nécessaire de prouver que le débiteur a commis une faute personnelle si ce dernier a introduit volontairement des substituts pour exécuter son obligation. L'acte fautif du substitut ne peut exonérer le débiteur. Pour Thérèse Rousseau-Houle, "l'admission d'une responsabilité contractuelledu faitd'autruic conduit àl'aggravation de la responsabilité des agences de voyages quipeuventen quelque sorte devenirgarants des fournisseurs de services. L'extension de la responsabilité des agences est certes la conséquence directe de l'évolution de leur rôle; pourtant la jurisprudence ne distingue pas toujours selon le rôle joué par les agences. A quel que titre que celles-ci agissent, on constate que nos tribunaux cherchent à protéger les consommateurs de sorte que la mise encause d'une agence de voyages aboutit presque inéluctablement à une indemnisation du préjudice subi par son client" ${ }^{\prime 27}$.
$\mathrm{Si}$, en règle générale, l'extension de la responsabilité des agences semble s'imposer, il faut néanmoins nuancer selon les faits générateurs de responsabilité: les fausses représentations ou l'absence de certaines informations, même celles qui sont le fait d'un fournisseur de services, engageront davantage la responsabilité des organisateurs de voyages, voire des intermédiaires que la perte des bagages subie par un voyageur lors du transport aérien. Les limites du présent article ne permettent pas de distinguer, mais Thérèse Rousseau-Houle en a fait un bon survol ${ }^{28}$.

\section{De nouvelles questions juridiques}

L'importance des systèmes de réservations informatisés, largement miseen valeur dans le présent numérode Téoros (voir les articles de Simier, Tremblay, Louillet, Rome) amène présentement le législateur fédéral à envisager l'adoption d'une loi pour régir l'industrie de ces systèmes. Le développement de ces systèmes est loin de respecter la saine concurrence, ce qui peut être néfaste autant pour les agences de voyages que pour les consommateurs. Les contrats entre les grands systèmes de réservations et les agences de voyages ou entre les transporteurs aériens, affiliés àces systèmes et les agences, peuvent avoir pour conséquence de biaiser l'offre de services, et même carrément de la réduire surcertaines destinations. Les agences n'ayant pas de prise sur l'information dispensée par les systèmes informatisés, la responsabilité découlant d'une information erronée ne devrait-elle pas être attribuée au système de réservations plutôtqu'à l'agence? Ces deux problèmes illustrent les questions que pourrait résoudre la future législation. Récemment, ACTA-Canada soumettait un mémoire sur la future loi et suggérait diverses mesures complémentaires tout en appuyant le principe de réglementer ce domaine ${ }^{29}$. Cet exemple rappelle que le droit n'est pas figé et que l'évolution de la technologie pose de nouveaux problèmes qu'il faut tenter de régler dans le respect d'une saine concurrence commerciale, toujours de plus en plus difficile à maintenir face à de grandes entreprises monopolistiques, et dans le respect du consommateur. Le droit réagit en retard, souvent timidement...mais c'est le dernier recours pour contrer les abus.

\section{Notes explicativas}

(1) Loi sur les agents de voyages, L.R.O. c. A-10

(2) Reglement sur les agents de voyages, R.R.O. 1981 . c. A-10, r. 1 (modifié)

(3) Réglemient modifiant le règlement sur les agents de voyages, Decret 449-90, 4 avril 1990, Gazette officlelle du Quebbec, 11 avril 1990, p. 1063

(4) Une légère hausse dans le coùt du permis: pour les détaillant, le coút est fixé a 2255 au lieu de 2005 , pour les grossistes, 4505 au lieu de 4005 , etc. Une hausse importante des montants est à souligner pour le cautionnement individuel. Les régles sont plus strictes concernant les retraits du compte en fiducie, de nouvelles regles s'appliquent à la publicité faite par un agent de voyages (mention des sommes a payer en sus du coút annoncé, dimension des caractéres typographiques dans le cas d'une publicité ecrite...1.

(5) Serge LAMARAE, Les agences de vovages sont les pires délinquants soion les chiffres de $\mathrm{POPC}$. Tourisme Plus, no.316,13-26 août 1990, p.20.

(6) Loi sur la protectiondu consommateur, L.R.Q., C. P-40.1; cette loi est complétée par le Règlement d'application de la Loi sur la protection du consommateur, R.R.0. 1981, c. P-40.1 r. 1 .

(7) Comartin c. Bordet, [1984] C, S. 584.

(8) Foland-Yves GAGNE, Le droit des agences de voyages et le transport aérien, Montréal, Milson 8 Lafleur/Screj, 1982, 170p.

Roland-Yves GAGNE, La responsabilite des difforents intervenants en matiere de vovage, [1989) 49 R. de B. $521-558$.

(9) Thertese AOUSSEAU-HOULE, Leregimejuridique des agences de voyages, (1976) 17 C. de D. 353 371.

Therese ROUSSEAU-HOULE, La responsabulite des agences de voyages: les tendances de la Murisprudence récente, (1983) 24 C. de D. 643. 671.

(10) Parmiles auteurs français cités: Pierre COUVRAT, Les agences de voyages en droit francais, L.G.D.J., 1967 p. 1 et ss:; R, RODIERE, La responsabilité des agences de voyages, D. 1958.249 et $Y$ a-t-il une responsabilité contractuelie du fait d'autrui, D. 1952.79.

Fucemment, un nouvel auteur français a fait le point sur cette question. II s'agit de Plerre PY. Drolt du tourisme, Paris, Dalloz, 1989, $340 \mathrm{p}$. (surtout $\rho p$. $217-250$ ) et $L a$ responsabulité contractuelle des intermediaires de vovages et des autres professionnels du tourigme : ombres et fumieres, Tourisprudence, no 1. Paris, ETE, juin 1990, pp. 19-40.

(11) Therèse ROUSSEAU.HOULE, La responsabilit des agences de voyages: les tendances de la jurisprudence récente, op. cit., p. 644

(12) Entre autres: Dovan c. Demetelin, [1980| C.P. 234: Aoussersu C. Agence de vorages en hberté Quebec inc., C.P. Quebec 200-02-04229-771, le 19/12/1978 (J.E. 79-139)

(13) Ste Marie c. Voyage Montabault inc. [1979] R.L., 583 [C.P.].

(14) Vincell c. Boshi, C.P. Montresal 500-02-052878803. le 5/4/1982 (J.E. 82-473) Rousseav c. Agence do wovages en liberte Ouébec inc. Idéjá cité).

(15) Audet c. Carbeil, [1975] R.L, 320 (C.P.)

(16) Deschesnes c. Juan, |1975) R.L. 267 (C. P)

(17) Aubuchon c Bourderan, [1976] C.P. 537

(18) Ethel GROFFIER, L'importance du mandat en droit qudbeccis: les développements rúcents, (1\$85) 15 R.D.U.S., Pp. 483-484

(19) Foland-Yves GAGNE, La responsabilité des differents intervenants en matiere de vovages, cp, cit , pp.535-536.

(20) Ibid, p. 536 .

(21) Déja cité, voir note 7

[22) Mercier c. Vovages Frontenac Inc, [1977] R.L. 547 (C.P.).

(23) Pétrinc. Voyages P.L.M. Inc. C.P. Montréal 500 02-008210-820, le 27/6/1983 (J.E. 83-793)

(24) Burns c. Vocances Camival (Ouébec) Ltée, |1986| R.J.O., pp. 943945.

(25) Ibid, p 944945

(26) Gosselin c. Service de voyages Kues Bordeleau Inc. C. . Trois Rivieres 400-02-000788-883. Ie 18/4/1990 (J.E. 90-922)

(27) Therese ROUSSEAU-HOULE, La responsabulite des agences de woyages: les tendances de la jurisprudence récente, op. cit, p. 671.

(28) Ibid

(29) Serge LAMARAE, LACTA se penche sur la loi des systemes die réservations informatisés. Tourisme Plus ,no.317, 21 au 27 mai 1990, p.8. 